\title{
Analyzing Cardiovascular Variabilities in Patients with Heart Failure
}

\author{
A Voss ${ }^{1}$, R Schroeder ${ }^{1}$, M Baumert ${ }^{1}$, S Truebner ${ }^{1}$, M Goernig ${ }^{2}$, A Hagenow ${ }^{3}$, HR Figulla ${ }^{2}$ \\ ${ }^{1}$ University of Applied Sciences Jena, Department of Medical Engineering, Germany \\ ${ }^{2}$ Friedrich Schiller University Jena, Clinic of Internal Medicine I, Germany \\ ${ }^{3}$ Center of Internal Medicine Elsterwerda, Germany
}

\begin{abstract}
Nearly $60 \%$ of patients with heart failure (HF) suffer from cardiac death within five years after diagnosis. Strategies for early diagnosis of CHF are rather insufficient. Therefore, the objective of this study was to develop a parameter set for an enhanced risk stratification in $\mathrm{HF}$ patients. From 43 patients suffering from HF (NYHA $\geq I I$, $E F<45 \%)$ and from 20 healthy subjects (REF) heart sound (HS), heart rate and blood pressure variability (HRV and BPV), interactions between heart rate and blood pressure (joint symbolic dynamics - JSD) and blood pressure morphology (BPM) were analyzed. Measures from $B P V, B P M$ and $J S D$ revealed high significances $(p<0.0001)$ discriminating REF and HF. A set of three parameters from HS, JSD and BPM was developed (sensitivity $=91.7 \%$, specificity $=93.3 \%)$ for risk stratification in patients with heart failure.
\end{abstract}

\section{Introduction}

Nowadays, about 14 million Europeans are suffered from heart failure (HF). Statistical analyses forecast an increase of patients with $\mathrm{HF}$ to 30 million by the year 2020. Nearly $60 \%$ of HF patients suffer from cardiac death within five years after diagnosis. Furthermore, HF is the most common reason for hospitalization in the over-65 age group [1].

$\mathrm{HF}$ is a complex cardiovascular disease often resulting from any functional or structural cardiac disorder that is mostly caused by coronary artery disease, hypertension and cardiomyopathy and characterized by impaired ventricular filling or reduced ventricular ejection fraction (EF) [2]. The extent of HF by physicians is often assessed according to the New York Heart Association (NYHA) functional classification system that places patients in the categories NYHA I-IV based on how much they are limited during physical activity. However, currently applied strategies for early diagnosis of $\mathrm{HF}$ are not sufficient. An improved risk stratification of HF is necessary to assess the prognosis of $\mathrm{HF}$ and to find an adapted drug treatment or an optimal timing for pacemaker or cardioverter defi- brillator implantation or for heart transplantation.

The aim of this study was to develop a multivariate parameter set for an enhanced risk stratification in patients with heart failure.

\section{Methods}

Within this study, 43 patients suffered from $\mathrm{HF}$ characterized by $\mathrm{NYHA} \geq 2$ and $\mathrm{EF}<45 \%$ and 20 healthy subjects as reference (REF) were enrolled.

30 minutes of ECG and synchronized continuously blood pressure (NIBP) were recorded under standardized resting conditions (supine position, quiet environment, same time and place) using the non-invasive Portapres M2 blood pressure monitor (TNO-TPD, Amsterdam, Netherlands). Thereafter, heart sound and synchronized ECG as well as NIBP were acquired on 9 auscultation areas over 5 heart beats during the patient hold his breath. For the recording of heart sound a hand-held electronic stethoscope (Welch Allyn ${ }^{\circledR}$ Master Elite Plus Stethoscope) was applied. Heart sound were digitized using a high quality USB soundcard (Maya EX, Audiotrak, sampling frequency $44100 \mathrm{~Hz}$, resolution 16 bit). Using a commercially available amplifier system (Twente Medical Systems, Netherlands) ECG and NIBP were sampled with $\mathrm{f}_{\mathrm{s}}=1600 \mathrm{~Hz}$. After discretization the signals were stored in a data base together with the patient data. The diagnosis of all HF patients were confirmed by an experienced cardiologist using short- and long-term ECG as well as stress ECG, echocardiography and heart catheter examination.

From the 30 minute data records the time series of heart rate (tachogram) consisting of beat-to-beat intervals (BBI) and of blood pressure (systogram/diastogram) were extracted. Ectopic beats as well as other disturbances were removed. To quantify the heart rate variability (HRV) and blood pressure variability (BPV) several parameters of time domain, frequency domain and nonlinear dynamics were calculated from every tachogram, systogram and diastogram.

From time domain the following parameters [3] were calculated: 
- $\quad$ meanNN = mean value of BBI time series [ms];

- $\quad$ sdNN =standard deviation of BBI time series [ms];

- rmssd = square root of the mean squared differences of successive beat-to-beat intervals [ms];

- sys_meanNN, dia_meanNN = mean systolic and diastolic blood pressure $[\mathrm{mmHg}]$;

- sys_sdNN, dia_sdNN = standard deviation of the systolic and diastolic blood pressure [mmHg];

- sys_rmssd, dia_rmssd = square root of the mean squared differences of successive systolic and diastolic blood pressure intervals $[\mathrm{mmHg}]$.

Power spectra of the time series were estimated using the Fast Fourier transform (Blackman Harris window) and frequency domain parameters were computed:

- ULF, sys_ULF, dia_ULF = power in the frequency band $0-0.0033 \mathrm{~Hz}\left[\mathrm{~s}^{2}\right]$;

- VLF, sys_VLF, dia_VLF = power in the frequency band $0.0033-0.04 \mathrm{~Hz}\left[\mathrm{~s}^{2}\right]$;

- LF, sys_LF, dia_LF = power in the frequency band $0.04-0.15 \mathrm{~Hz}\left[\mathrm{~s}^{2}\right]$;

- HF, sys_HF, dia_HF = power in the frequency band $0.15-0.4 \mathrm{~Hz},\left[\mathrm{~s}^{2}\right]$;

- P, sys_P, dia_P = total spectral power $\left[\mathrm{s}^{2}\right]$.

Furthermore, the measures of normalized LFn, HFn as well as the ratios LF/HF, LF/P and HF/P were calculated.

To classify dynamic changes within time series the following parameters from nonlinear symbolic dynamics [4] were estimated:

- plvar2, plvar5, plvar20 = portion of low-variability patterns within the BBI time series $<2 \mathrm{~ms},<5 \mathrm{~ms}$, $<20$ ms over 6 heart cycles

- phvar2, phvar5, phvar20 = portion of highvariability patterns within the BBI time series $>2 \mathrm{~ms},>5 \mathrm{~ms},>20 \mathrm{~ms}$ over 6 heart cycles

- sys_ plvar2, sys_plvar5, sys_plvar20 and dia_plvar2, dia_plvar5, dia_plvar20 = portion of low-variability patterns in the systolic or diastolic blood pressure $<2 \mathrm{mmHg},<5 \mathrm{mmHg},<20 \mathrm{mmHg}$;

- sys_phvar2, sys_phvar5, sys_phvar20 and dia_phvar2, dia_phvar5, dia_phvar20 = portion of high-variability patterns in the systolic or diastolic blood pressure $>2 \mathrm{mmHg},>5 \mathrm{mmHg},>20 \mathrm{mmHg}$.

To analyze the interactions between BBI and systolic blood pressure (SP) the baroreflex sensitivity (BRS) was estimated by using the dual sequence method [5]. Thereby the slope of the linear regression function between monotonous dropping SP sequences followed by monotonous shortening BBI sequences of three successive beats were calculated.

A further nonlinear measure, the joint symbolic dynamics (JSD), was applied to describe the nonlinear interactions between BBI and SP by means of symbols [6]. Thereby, both time series were transformed into symbol sequences of zeros (negative slope) and ones (positive slope) and words of three successive symbols were formed. The normalized probabilities of all single word types $(\mathrm{n}=64)$ occurrences were computed (JSD1-JSD64) using an $8 \times 8$ word distribution density matrix (columns = BBI words and rows $=$ SP words) from word type $[000,000]^{\mathrm{T}}$ to $[111,111]^{\mathrm{T}}$. Furthermore the probabilities of the words within the BBI time series (BBI000 to BBI111) as well as within the SP series (SP000 to SP111) were determined. BBI010 and BBI101 are measures for the occurrence of alternans [7],[8].

Considering the blood pressure morphology (BPM) several parameters describing amplitudes, time intervals and slopes as well as areas from the blood pressure waveform were estimated [9] according to Figure 1.

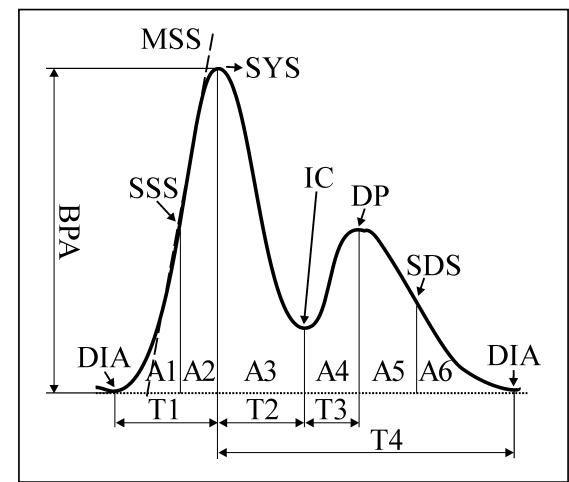

Figure 1. Parameters of blood pressure morphology. SYS: endsystolic blood pressure; DIA: enddiastolic blood pressure; BPA: blood pressure wave amplitude; SSS: maximum systolic slope; MSS: mean systolic slope; SDS: maximum diastolic slope; IC: incisure; DP: dicrotic peak; T1-T4: time intervals; A1-A6: areas under the curve.

For every heart beat the location and amplitude of the systolic (SYS) and enddiastolic blood pressure (DIA), dicrotic peak (DP) and incisure (IC) were determined using a correlation based algorithm. Blood pressure wave amplitude (BPA) was estimated as difference between SYS and DIA. Applying a linear regression algorithm the maximum and mean systolic slope (SSS, MSS) were computed between SYS and previous DIA and the minimum diastolic slope (SDS) was calculated between DP and DIA. At last, some time intervals (T1 - T4), several areas (A1 - A6) under the blood pressure curve and BBI normalized durations from the R wave to SYS (RSYS), DIA (RDIA) and Incisur (RINC) were estimated.

Finally, parameters that describe the first (frequency range: $5-100 \mathrm{~Hz}$ ) and second heart sound $(100-150 \mathrm{~Hz})$ were calculated using a wavelet based heart sound analysis method [10]. At first, for every patient and auscultation area BBI related heart sound segments were decomposed into frequency scale 6 (frequency range: $0-172 \mathrm{~Hz}$ ) that is suitable for the analysis of the first and second 
heart sound. For decomposition a multiresolution wavelet analysis, published by Mallat 1989 [11] using Daubechies wavelets of order 14 was applied. Noisy heart sound segments or any audible disturbance were excluded. The temporal locations of the first and second heart sound were calculated from every wavelet filtered signal using a Shannon energy and short-time Fourier transform (STFT) technique approach [12]. For every patient the first and second heart sounds were BBI normalized and averaged (HS1 and HS2) within the recorded auscultation areas.

After a 6-month follow-up the HF group was divided into two subgroups: $\mathrm{HF}$ with no progression $\left(\mathrm{HF}_{\mathrm{LR}}, \mathrm{n}=21\right.$, low risk group) and with significant progression of the disease including 7 patients suffered from cardiac death $\left(\mathrm{HF}_{\mathrm{HR}}, \mathrm{n}=22\right.$, high risk group). Mann-Whitney U-test $(\mathrm{p}<0.05)$ was assessed to find out whether the applied methods could differentiate between the two age and gender matched groups $\mathrm{HF}^{*}$ and $\mathrm{REF}^{*}$ (Table 1) as well as between $\mathrm{HF}_{\mathrm{LR}}$ and $\mathrm{HF}_{\mathrm{HR}}$ (Table 2). Considering the multiple testing problem we used corrected significance levels according to Bonferroni. Cox regression analysis were applied to find a multivariate parameter set for an optimal discrimination between $\mathrm{HF}_{\mathrm{LR}}$ and $\mathrm{HF}_{\mathrm{HR}}$.

Table 1. Group description $\mathrm{HF}^{*}$ and REF*: $\mathrm{n}$ - number of patients, EF - ejection fraction, NYHA - New York Heart Association functional class, Gender: 0-male, 1-female.

\begin{tabular}{|l|c|c|}
\hline group & HF$^{*}(\mathbf{n}=\mathbf{1 0})$ & REF $^{*}(\mathbf{n}=\mathbf{1 0})$ \\
\hline age & $53.28 \pm 8.15$ & $47.49 \pm 10.05$ \\
\hline gender & $0.36 \pm 0.51$ & $0.60 \pm 0.52$ \\
\hline EF & $30.18 \pm 7.64$ & - \\
\hline NYHA & $2.45 \pm 0.72$ & - \\
\hline
\end{tabular}

Table 2. Group description $\mathrm{HF}_{\mathrm{LR}}$ and $\mathrm{HF}_{\mathrm{HR}}$ : $\mathrm{n}$ - number of patients, EF - ejection fraction, NYHA - New York Heart Association functional class, Gender: 0-male, 1-female.

\begin{tabular}{|l|c|c|}
\hline group & $\mathbf{H F}_{\mathbf{L R}}(\mathbf{n = 2 1})$ & $\mathbf{H F}_{\mathbf{H R}}(\mathbf{n = 2 2})$ \\
\hline age & $64.36 \pm 10.69$ & $65.99 \pm 10.66$ \\
\hline gender & $0.29 \pm 0.46$ & $0.18 \pm 0.39$ \\
\hline EF & $39.43 \pm 15.37$ & $38.36 \pm 15.03$ \\
\hline NYHA & $2.48 \pm 0.56$ & $2.79 \pm 0.55$ \\
\hline
\end{tabular}

\section{Results}

Measures from BPV, BPM and JSD revealed high significances $(p<0.0001$, Bonferroni) discriminating the groups $\mathrm{HF}^{*}$ and $\mathrm{REF}^{*}$ but not from HRV, BRS and HS (Table 3). Normalized low frequency component (dia_LFn) as a marker of predominantly sympathetic modulation as well as the frequency ratio (dia_LF/HF) describing the sympathovagal balance were significantly higher within the $\mathrm{REF}^{*}$ group compared to HF*. The high frequency component (dia_HFn) as measure of the efferent vagal activity was significantly lower within the $\mathrm{REF}^{*}$ group. BPM parameters describing the systolic slopes (SSS, MSS) and the blood pressure wave amplitude (BPA) of the blood pressure curve were clearly decreased within $\mathrm{HF}^{*}$ compared to $\mathrm{REF}^{*}$. Furthermore, the BBI normalized interval between $\mathrm{R}$ wave and SYS (RSYS) and Incisur (RINC) were significantly different considering $\mathrm{HF}^{*}$ and $\mathrm{REF}^{*}$. Finally the occurrence of alternans (BBI010, BBI101) was significantly higher within $\mathrm{HF}^{*}$ in comparison to REF.

Table 3. BPV, BPM and JSD - significant $(\mathrm{p}<0.0001)$ parameters for discrimination between $\mathrm{HF}^{*}$ and $\mathrm{REF}^{*}$.

\begin{tabular}{|l|c|c|c|}
\hline parameter & HF $^{*}$ & REF* & p \\
\hline dia_LFn & $0.61 \pm 0.10$ & $0.92 \pm 0.03$ & 0.000006 \\
\hline dia_HFn & $0.39 \pm 0.10$ & $0.08 \pm 0.03$ & 0.000006 \\
\hline dia_LF/HF & $1.76 \pm 0.94$ & $13.18 \pm 6.95$ & 0.000006 \\
\hline BPA [mmHg] & $38.70 \pm 15.64$ & $67.19 \pm 7.37$ & 0.000068 \\
\hline SSS [mmHg/ms] & $0.62 \pm 0.27$ & $1.44 \pm 0.20$ & 0.000006 \\
\hline MSS [mmHg/ms] & $0.51 \pm 0.22$ & $1.05 \pm 0.15$ & 0.000068 \\
\hline RSYS & $0.35 \pm 0.04$ & $0.24 \pm 0.03$ & 0.000072 \\
\hline RINC & $1.50 \pm 0.07$ & $1.87 \pm 0.20$ & 0.000023 \\
\hline BBI010 & $0.21 \pm 0.05$ & $0.08 \pm 0.03$ & 0.000006 \\
\hline BBI101 & $0.19 \pm 0.05$ & $0.09 \pm 0.03$ & 0.000023 \\
\hline
\end{tabular}

To differentiate $\mathrm{HF}_{\mathrm{LR}}$ and $\mathrm{HF}_{\mathrm{HR}}$ patients at first univariate parameters were calculated (Table 4). NYHA was different in both groups $(\mathrm{p}=0.043)$. High risk patients were significantly more limited during physical activity as low risk patients. In contrast to the BPV analysis (sys_ULF/P, dia_VLP/P: $\mathrm{p}=0.047$ ) the short-term HRV analysis revealed no significant differences between $\mathrm{HF}_{\mathrm{LR}}$ and $\mathrm{HF}_{\mathrm{HR}}$. Blood pressure amplitude (BPA: $\mathrm{p}=0.03$ ) as well as diastolic slope (SDS: $\mathrm{p}=0.023$ ) were clearly decreased in $\mathrm{HF}_{\mathrm{HR}}$ patients. The probability of the word "011" within the BBI time series (BBI011: $\mathrm{p}=0.049$ ) was significantly higher for the $\mathrm{HF}_{\mathrm{LR}}$ group in comparison to $\mathrm{HF}_{\mathrm{HR}}$. From the heart sound analysis the normalized duration from the $\mathrm{R}$ wave to the first heart sound (HS1: $\mathrm{p}=0.022$ ) was obviously extended within $\mathrm{HF}_{\mathrm{HR}}$.

Table 4. Univariate significant parameters $(\mathrm{p}<0.05)$ for discrimination between $\mathrm{HF}_{\mathrm{LR}}$ and $\mathrm{HF}_{\mathrm{HR}}$.

\begin{tabular}{|l|c|c|c|}
\hline parameter & $\mathbf{H F}_{\mathbf{L R}}$ & $\mathbf{H F}_{\mathbf{H R}}$ & $\mathbf{p}$ \\
\hline NYHA & $2.48 \pm 0.56$ & $2.80 \pm 0.55$ & 0.043 \\
\hline sys_ULF/P & $0.25 \pm 0.18$ & $0.40 \pm 0.25$ & 0.047 \\
\hline dia_VLF/P & $0.51 \pm 0.19$ & $0.39 \pm 0.23$ & 0.047 \\
\hline BPA [mmHg] & $42.23 \pm 17.76$ & $31.89 \pm 21.04$ & 0.030 \\
\hline SDS [mmHg/ms] & $-0.49 \pm 0.26$ & $-0.34 \pm 0.09$ & 0.023 \\
\hline BBI011 & $0.13 \pm 0.03$ & $0.12 \pm 0.03$ & 0.049 \\
\hline HS1 & $0.10 \pm 0.03$ & $0.13 \pm 0.04$ & 0.022 \\
\hline
\end{tabular}


For risk stratification in differentiating the groups $\mathrm{HF}_{\mathrm{LR}}$ and $\mathrm{HF}_{\mathrm{HR}}$ an optimal set of three univariate significant parameters was determined leading to a sensitivity of $91.7 \%$ and a specificity of $93.3 \%$. This parameter set consists of linear and non-linear parameters from HS (HS1), BPM (SDS) and JSD (BBI011).

\section{Discussion and conclusions}

The applied methods for analyzing BPV, BPM, JSD and HS appear to be suitable for an enhanced diagnosis of HF including improved risk stratification. According to the MACAS study [13] analysis of short term HRV did not contribute to risk stratification. However, Nolan et al [14] investigated 433 patients suffered from congestive heart failure (CHF) and showed that a reduced SDNN yielded from 24-hour Holter ECG identifies patients at high risk of death due to progressive heart failure. Galinier et al [15] demonstrated a relation between reduced daytime LF power from 24-hour Holter recording and sudden death in patients with $\mathrm{CHF}$.

Within this study a highly significant discrimination between HF and REF $(\mathrm{p}<0.0001)$ was revealed. Furthermore, an optimal parameter set of three parameters (HS1, SDS, BBI011) with a specificity of $93.3 \%$ and a sensitivity of $91.7 \%$ was estimated and seems to be useful for a risk stratification in HF patients.

To validate the results of this study further prospective studies with an increased number of patients and a prolonged follow up period are necessary. A further study should investigate whether the combination with longterm HRV parameters further enhances risk stratification in HF patients.

\section{Acknowledgements}

This study was partly supported by grants from the Federal Ministry of Education, Science, Research and Technology BMBF (AiF 1706502).

\section{References}

[1] SHAPE (Study on Heart failure Awareness and Perception in Europe). Understanding Heart Failure. http://www.heartfailure-europe.com.

[2] Hunt et al.. ACC/AHA 2005 Guideline Update for the Diagnosis and Management of Chronic Heart Failure in the Adult. American College of Cardiology Foundation and the American Heart Association 2005. http://www.acc.org/clinical/guidelines/failure/index.pdf

[3] Task Force of the European Society of Cardiology and the North American Society of Pacing and Electrophysiology. Heart rate variability - Standards of Measurement. Physiological Interpretation and Clinical Use. Circulation 1996;93(5):1043-65.

[4] Voss A, Kurths J, Kleiner HJ, Witt A, Wessel N, Saparin P, Osterziel KJ, Schurath R, Dietz R. The application of methods of non-linear dynamics for the improved and predictive recognition of patients threatened by sudden cardiac death. Cardiovascular Research 1996;31:419-33.

[5] Malberg H, Wessel N, Schirdewan A, Osterziel KJ, Voss A. Dual sequence method for analysis of spontaneous baroreceptor reflex sensitivity in patients with dilated cardiomyopathy. Zeitschrift für Kardiologie1999;88(5):331-7.

[6] Baumert M, Walther T, Hopfe J, Stepan H, Faber R, Voss A. Joint symbolic dynamic analysis of beat-to-beat interactions of heart rate and systolic blood pressure in normal pregnancy. Med Biol Eng Comput 2002;40(2):241-5.

[7] Voss A, Baier V, Hopfe J, Schirdewan A, Leder U. Heart Rate and Blood Pressure Turbulence - Marker of the Baroreflex Sensitivity or Consequence of Postextrasystolic Potentiation and Pulsus Alternans?. Am J Cardiol 2002;89(1):110-1.

[8] Leder U, Pohl HP, Baier V, Baumert M, Liehr M, Haueisen J, Voss A, Figulla HR. Alternans of blood pressure and heart rate in dilated cardiomyopathy. Pacing Clin Electrophysiol 2002;25(9):1307-14.

[9] Faber R, Stepan H, Baumert M, Voss A, Walther T. Analysis of blood pressure waveform: a new method for the classification of hypertensive pregnancy disorders. Journal of Human Hypertension 2004; 18:135-7.

[10] Herold J, Schroeder R, Nasticzky F, Baier V, Mix A, Huebner T, Voss A. Diagnosing aortic valve stenosis by correlation analysis of wavelet filtered heart sounds. Med Biol Eng Comput 2005 (in press).

[11] Mallat SG. A theory for multiresolution signal decomposition. IEEE Transactions on Pattern Analysis and Machine Intelligence 1989;11(7):674-93.

[12] Voss A, Mix A, Huebner T. Diagnosing Aortic Valve Stenosis by Parameter Extraction of Heart Sound Signals. Annals of Biomedical Engineering 2005;33(9):1184-91.

[13] Grimm W, Christ M, Bach J, Mueller HH, Maisch B. Noninvasive arrhythmia risk stratification in idiopathic dilated cardiomyopathy: results of the Marburg Cardiomyopathy Study. Circulation 2003;108(23):2883-91.

[14] Nolan J, Batin PD, Andrews R, Lindsay SJ, Brooksby P, Mullen M, Baig W, Flapan AD, Cowley A, Prescott RJ, Neilson JMM, Fox KAA. Prospective Study of Heart Rate Variability and Mortality in Chronic Heart Failure - Results of the United Kingdom Heart Failure Evaluation and Assessment of Risk Trial (UK-Heart). Circulation 1998;98:1510-6.

[15] Galinier M, Pathak A, Fourcade J, Androdias C, Curnier D, Varnous S, Boveda S, Massabuau P, Fauvel M, Senard JM, Bounhoure JP. Depressed low frequency power of heart rate variability as an independent predictor of sudden death in chronic heart failure. Eur Heart J. 2000; 21: 475-82.

Address for correspondence

Prof. Dr.-Ing. A. Voss

University of Applied Sciences

Department of Medical Engineering

Carl-Zeiss-Promenade 2

07745 Jena

voss@fh-jena.de 